\title{
Some New Findings in the Liquid Crystals of Sodium Salt of Deoxyribonucleic Acid
}

\author{
Eisaku IızuKa \\ Institute of High Polymer Research, Faculty of Textile Science and Technology, \\ Shinshu University, Ueda 386, Japan.
}

(Received November 22, 1976)

\begin{abstract}
The concentrated solutions of sodium salt of deoxyribonucleic acid have been observed under crossed polaroids and tested by means of the small-angle laser light scattering, the ultraviolet and infrared absorptions, the X-ray diffraction, the circular dichroism, and some other physical methods. The molecules of this polynucleotide aggregate to form rod-like assemblies (molecular clusters) at a certain polymer concentration and eventually form liquid crystalline structures. These liquid crystals are very similar to those found in the concentrated aqueous solutions of deoxygenated sickle-cell hemoglobin or of sodium salt of poly(glutamic acid).
\end{abstract}

KEY WORDS Deoxyribonucleic Acid / Lyotropic Liquid Crystal / Molecular Cluster /

The liquid crystals of living systems are naturally found in aqueous medium and the study of lyotropic liquid crystals of biopolymers with water is expected to supply useful information concerning the structure and functions of living systems. In the concentrated solutions of deoxyribonucleic acid (DNA), Robinson ${ }^{1}$ observed the twisted structure (cholesteric structure) that resembled the structure ${ }^{2}$ found in the concentrated solutions of poly( $\gamma$-benzyl L-glutamate) (PBLG) with some organic solvents. This was the first study of the liquid crystals of biopolymers. Spencer ${ }^{3}$ and his coworkers was able to observe such twisted structure also in transfer ribonucleic acid (tRNA) from baker's yeast. Quite recently, the author reported on the liquid crystals of deoxygenated sickle-cell hemoglobin (deoxyHb S) ${ }^{4}$ and of sodium salt of poly(glutamic acid) (PGA-Na) ${ }^{5}$, each found in aqueous solution. These liquid crystals are very similar to those of polypeptides such as PBLG and $\operatorname{poly}\left(\gamma\right.$-ethyl L-glutamate) (PELG) ${ }^{6}$ except that the viscosity of the solution increases markedly upon the liquid crystal formation; these polymer molecules aggregate to form rodlike assemblies or assemblies that become rodlike very easily under shearing stresses. In this paper, the author will report some new findings in the liquid crystals of sodium salt of DNA (NaDNA).

\section{EXPERIMENTAL}

\section{Material}

The NaDNA was purchased from Sigma Chemical Co. (Type III, highly polymerized from salmon sperm), and used directly without further purification. The polymer was dissolved in deionized water with $0.1 \mathrm{~mol} \mathrm{NaCl}(\mathrm{pH} \mathrm{7.0)}$ ) and was introduced in a quartz cell of path length $1 \mathrm{~mm}$. A quartz spacer was then inserted into the optical cell to shorten the path length to about $0.1 \mathrm{~mm}$ or less. The $\mathrm{pH}$ of the solution was not readjusted, and the polymer concentration was determined spectrophotometrically, assuming that the molar extinction coefficient of DNA is $6500 \mathrm{~mol}^{-1} \mathrm{~cm}^{-1}$ at $260 \mathrm{~nm}^{7}$

\section{Methods}

Polarizing micrographs of the solution were taken under crossed polaroids, and low-angle light-scattering patterns were photographed with a 2-mW $\mathrm{He}-\mathrm{Ne}$ gas laser (having 6328-A light) as the light source. The ultraviolet (UV) linear dichroism was measured with the UV attach- 

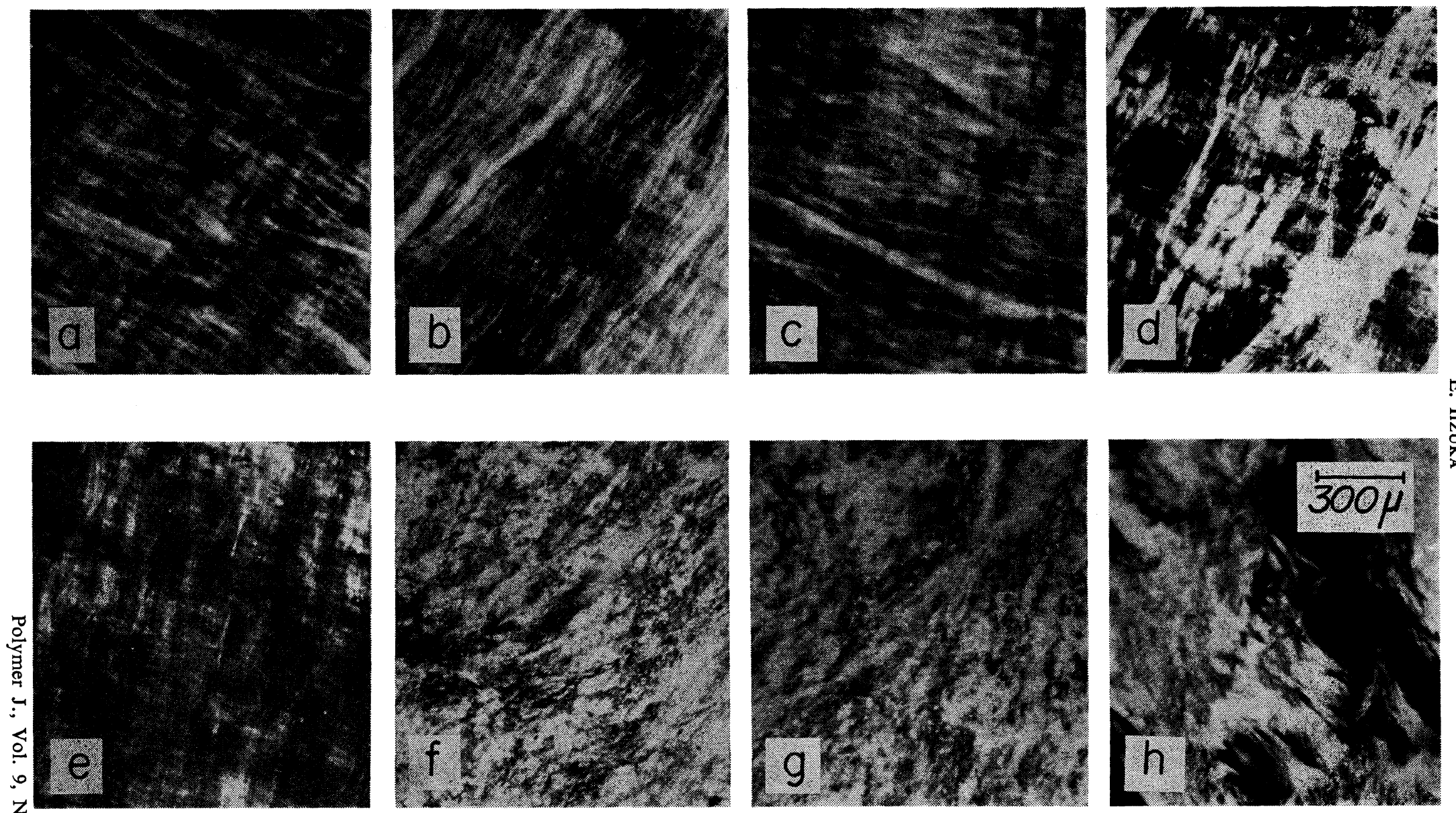

Figure 1. Polarizing micrographs of the concentrated solutions of NaDNA. Polymer concentration in $\%(w / w): a, 4.2 ; b$ and $c, 6.7 ; d$ and $e$, 8.4; $\mathrm{f}, 16.8 ; \mathrm{g}, 25.2 ; \mathrm{h}, 75.0$. The solution was given shearing stresses vertically in micrograph "e". 


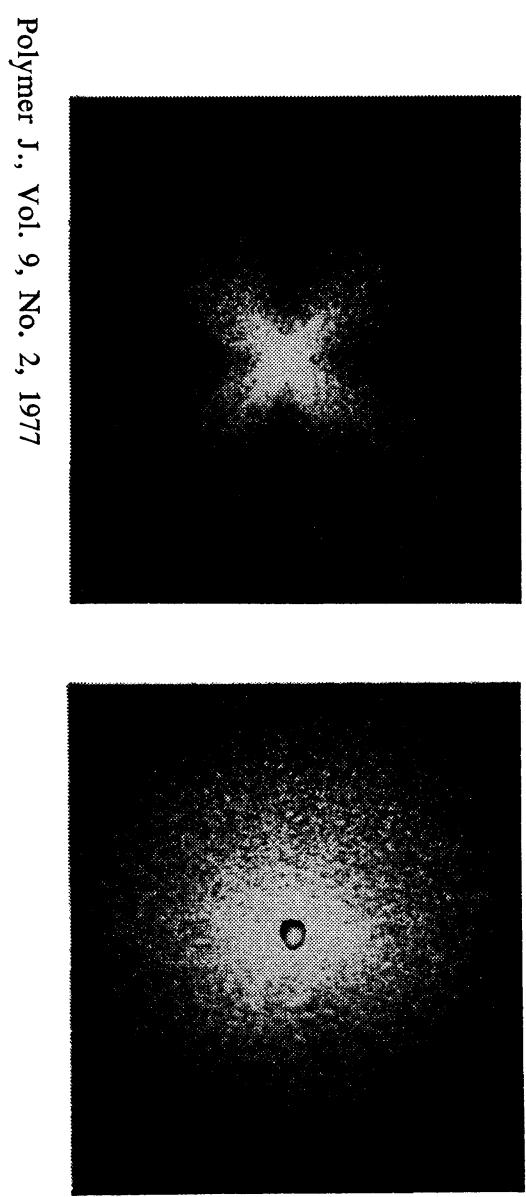

a
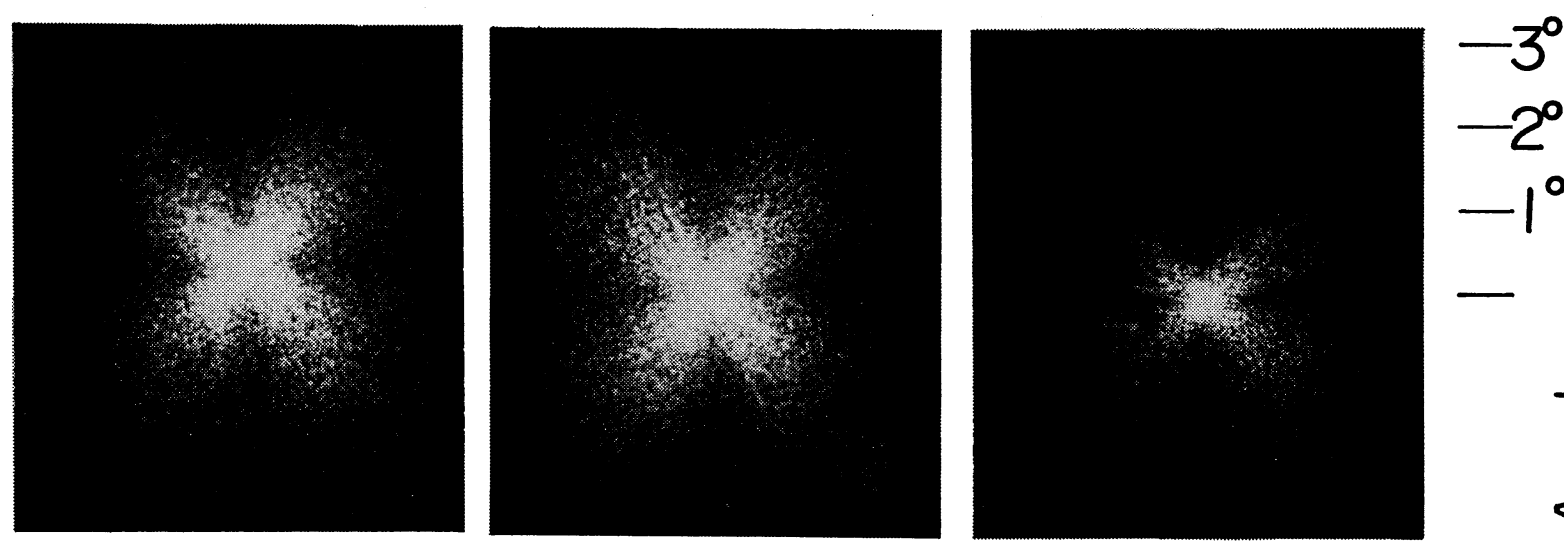

$-2^{\circ}$

$-1^{\circ}$
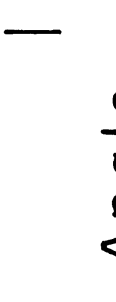

品

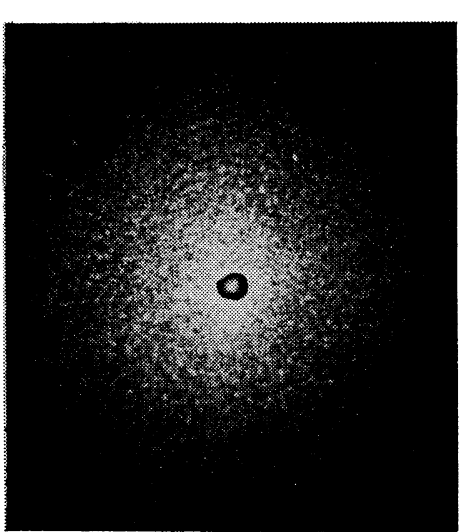

b

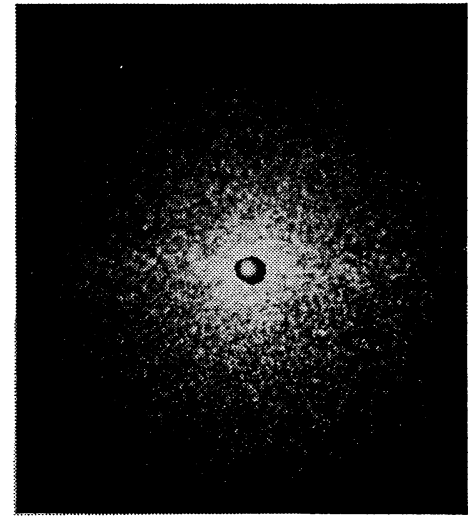

C

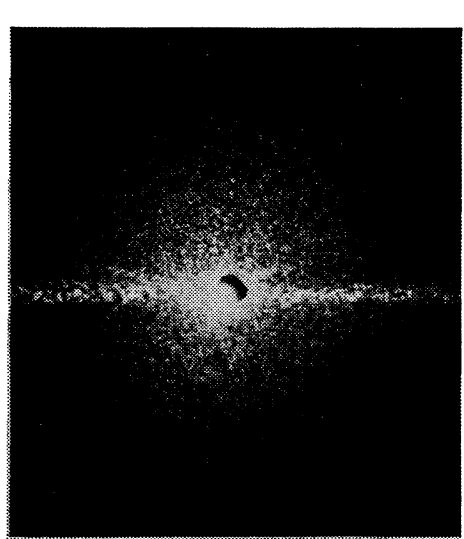

d

Figure 2. Small-ange laser-light-scattering patterns of the concentrated solutions of NaDNA. Upper, the $H_{\nabla}$ patterns; lower, the $V_{v}$ patterns. Polymer concentration in $\%(w / w): a, 3.4 ; b$ and $c, 6.7 ; d, 8.4$. Patterns "c" correspond to Figure 1c and patterns "d" to Figure 1e. 
ment of a Jasco ORD/UV-5 spectropolarimeter. The solution prepared with $\mathrm{D}_{2} \mathrm{O}$ was sandwiched between two $\mathrm{CaF}_{2}$ plates and the infrared (IR) linear dichroism was also measured with a Jasco DS-301 spectrophotometer. In these dichroism measurements, orientation of the solution was obtained by shifting the spacer or by moving the one plate with respect to the other. The solution was gel-like and orientation of the solution was kept during the measurements. With a Rigaku Denki geigerflex $\left(35 \mathrm{kV}, 25 \mathrm{~mA}, \mathrm{Cu}-\mathrm{K}_{\alpha}\right.$ line), X-ray diffraction photographs were taken in a glass capillary tube of diameter $1 \mathrm{~mm}$. The optical cell was mounted on a cell holder that can be rotated in a plane perpendicular to the incident beam and circular dichroism (CD) measurements were carried out with the Jasco spectropolarimeter. The measurements were carried out at out room temperature.

\section{RESULTS AND DISCUSSION}

The solution of NaDNA started to become slightly tubid at a certain polymer concentration below $1 \%(\mathrm{w} / \mathrm{w})$ and gradually became gel-like. It was already birefringent at $1 \%$; however, the change was so gradual that the exact concentration for the isotropic-to-birefringent transition could not be determined. The measurements were carried out on the solutions in the birefringent state.

\section{Observation under Crossed Polaroids}

Narrow, parallel lines that are different from those typical of the cholesteric structure are seen almost in one direction (Figure 1a-e) and forms a large domain that sometimes covers most of the area under the microscopic stage. The pattern looks fiber-like in some ways and suggests that the double-stranded molecules of $\mathrm{DNA}^{8}$ are oriented mainly due to the shearing stresses caused by the insertion of the spacer into the optical cell. We have been unsuccessful in preparing solutions whose structure is not affected by the shearing stresses. This pattern is seen especially when the polymer concentration is $8.4 \%$ or less, and is very similar to those seen in the oriented liquid crystals of deoxyHb $\mathrm{S},{ }^{4}$ of PGA-Na ${ }^{5}$, and of some polypeptides such as $\mathrm{PBLG}^{9}$ and $\mathrm{PELG}^{10}$ in organic solvents. At polymer concentrations of 16.8 and $25.0 \%$, the direction of the lines is no longer uniform (Figure 1f and g). This suggests a change of the structure in the birefringent solution, and at $75 \%$ the patterns appears to show the formation of some crystalline structure (Figure 1h).

\section{Small-Angle Laser Light Scattering}

For the solutions whose polymer concentrations are $8.4 \%$ or less, the $\mathrm{H}_{v}$ pattern (the one taken with the polarizer placed vertically and the analyzer horizontally) is always of the $\pm 45^{\circ}$ type (Figure 2), suggesting that the solutions are a collection of rod-like assemblies (molecular clusters) of the polymer molecules in which the maximum polarizability directions are parallel or perpendicular to their (long) rod axes. ${ }^{9,11}$ When the solution is placed so that the lines (that should be seen under crossed polaroids) are horizontal, the upper (and the lower) included angle of the cross pattern (the $\pm 45^{\circ}$ pattern) is less than $90^{\circ}$ and the $V_{v}$ pattern (the one taken with both the polarizer and the analyzer placed vertically) has a tendency to be long lengthwise; when these lines are vertical, both the $H_{v}$ and $V_{v}$ patterns tilt by $90^{\circ}$. These are the indications that the rod-like molecular clusters are aligned in the direction parallel to the lines. $^{4,9}$ This has been the case in the liquid crystals of deoxyHb S, ${ }^{4}$ of PGA-Na, ${ }^{5}$ and of some polypeptides such as $\mathrm{PBLG}^{9}$ and $\mathrm{PELG}^{10}$ in organic solvents.

The solution begins to scatter light irregularly at a polymer concentration of about $12 \%$ and the cross pattern becomes unable to be observed at polymer concentrations of $16.8 \%$ or more. This suggest some continuous change in the solution structure with the polymer concentration, in which multiple scattering of the incident light will be involved, and this is consistent with the observation under crossed polaroids.

\section{Linear Dichroism and Linear Birefringence}

At one of the main UV bands $(260 \mathrm{~nm})$, the birefringent solution of NaDNA shows a stronger absorption when the electric vector of the incident beam is perpendicular to the lines (to be seen under crossed polaroids) and a weaker absorption when the vector is parallel to the lines, although the difference is small, and so do some IR bands (Figure 3). The $260-\mathrm{nm}$ band is due to the $\pi-\pi^{*}$ transition in the purine and pyri- 


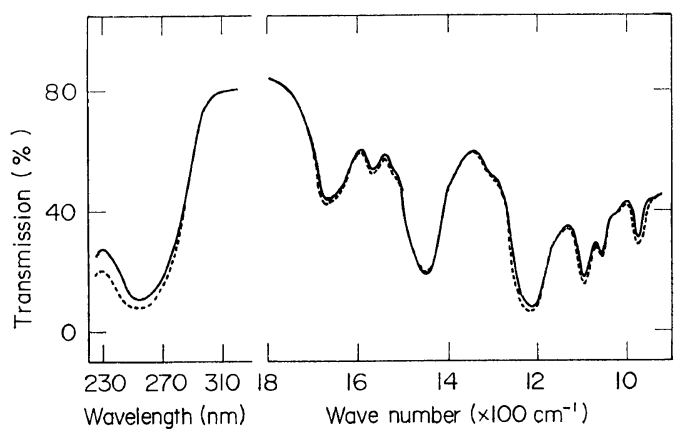

Figure 3. Ultraviolet and infrared absorption spectra of the concentrated solutions of NaDNA. The electric vector of the incident beam is parallel (solid lines) or perpendicular (broken lines) to the orientation direction. The ultraviolet spectra were measured in $\mathrm{H}_{2} \mathrm{O}$ and the infrared spectra in $\mathrm{D}_{2} \mathrm{O}$.

midine bases of the DNA molecules and the direction of the transition is in the planes of these bases. ${ }^{12,13}$ These planes are nearly perpendicular to the fiber axis of the double-stranded DNA molecules. ${ }^{8}$ Therefore, the dichroism result obtained suggests that the molecules are aligned parallel to the lines. The dichroism of several IR bands centered at 1680, 1210, 1090, and $970 \mathrm{~cm}^{-1}$ has the same sense (perpendicular dichroism) as that of the corresponding bands of the oriented films of DNA, ${ }^{14}$ although the dichroism is weaker in the birefringent solution. This dichroism result supports the idea concerning the direction of DNA molecules with respect to the direction of the lines. It will now be clear that the maximum polarizability direction of the rod-like molecular cluster is perpendicular to its long rod axis.

The oriented solution shows the negative birefringence as well as the negative polarization dichroism. The magnitude of the birefringence divided by the volume fraction of the polymer increases with the polymer concentration (Table I); however, the maximum value obtained is only 0.0020 and is far smaller than that of the

Table I. Birefringence of the oriented solutions of NaDNA

\begin{tabular}{cccc}
\hline $\begin{array}{c}\text { Polymer } \\
\text { concentration, } \\
\%(\mathrm{w} / \mathrm{w})\end{array}$ & 5.0 & 6.7 & 8.4 \\
\hline$-\left(\Delta n / c_{\mathrm{V}}\right)_{547 \mathrm{~nm}}$ & 0.0014 & 0.0016 & 0.0020 \\
\hline
\end{tabular}

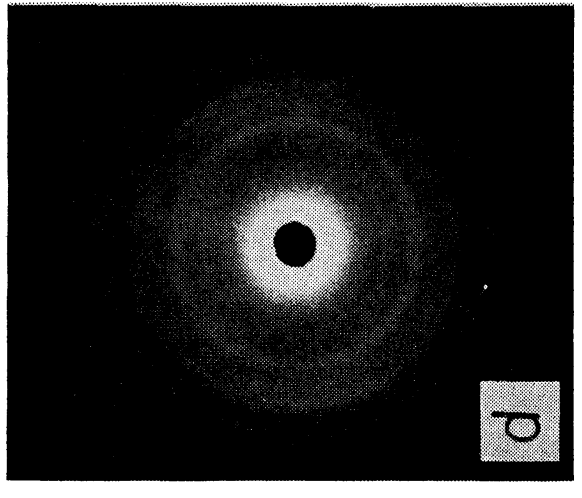

กิ่

ก
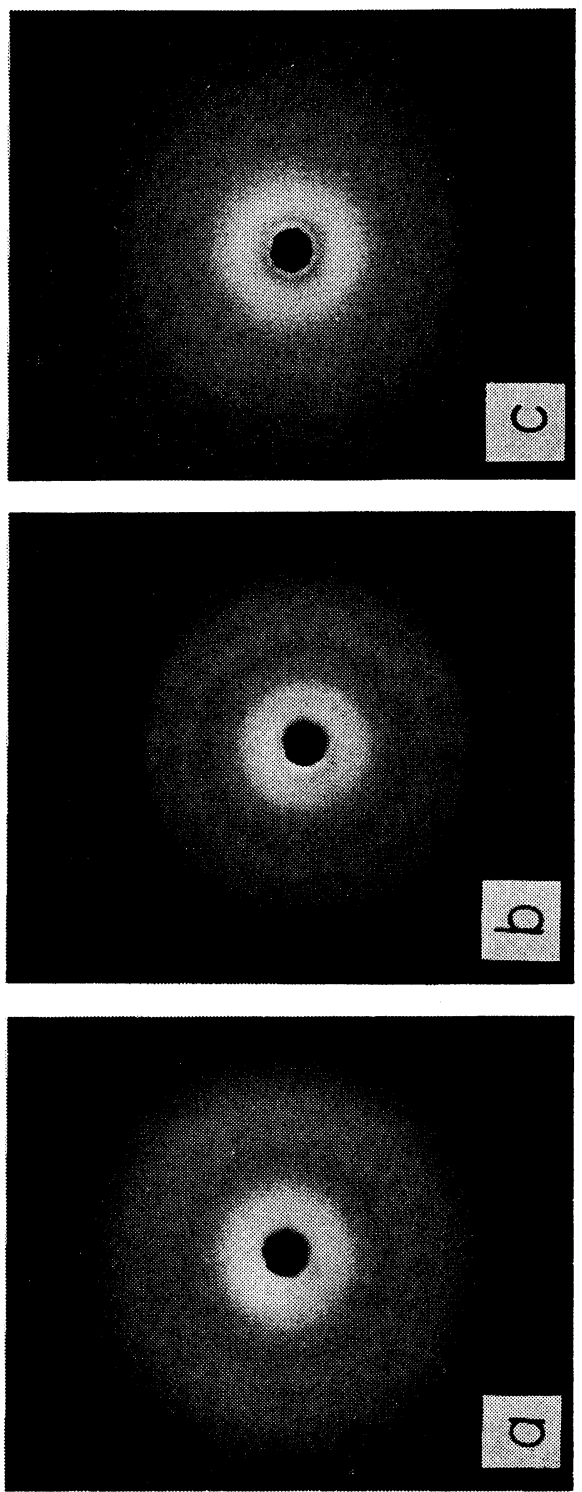

$\dot{2}$ ดे

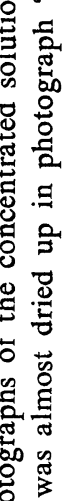
늘 동 
Table II. Lattice spacings of the birefringent solutions of NaDNA

\begin{tabular}{ccccc}
\hline $\begin{array}{c}\text { Polymer } \\
\text { concentration, } \\
\%(\mathrm{w} / \mathrm{w})\end{array}$ & 8.4 & 25.2 & 50.4 & 75.0 \\
\hline Spacing A, A & $3.5_{1}$ & $3.5_{1}$ & $3.4_{8}$ & $3.3_{8}$ \\
Spacing B, A & $4.4_{8}$ & $4.4_{0}$ & $4.3_{4}$ & $4.3_{0}$ \\
\hline
\end{tabular}

DNA crystal (0.056). ${ }^{15}$ Judging from the distortion of the shape of the $\pm 45^{\circ}$ pattern (Figure $2 d)$, the orientation of the rod-like molecular clusters should not be so poor. ${ }^{9}$ Therefore, this birefringence result (together with dichroism result) suggest that the ordering of the polynucleotide molecules is poor or/and these molecules fluctuate in each molecular cluster.

\section{$X$-Ray Diffraction}

Several Debye-Scherrer rings are observable in the X-ray diffraction photographs of the birefringent solutions of NaDNA. They become less broad with increasing polymer concentration (Figure 4), and it is known that two main lattice spacings (3.4 and 4.4 A) decrease accordingly (Table II). The corresponding two rings arise from axial periodicities of the double-stranded DNA molecules, and the 3.4-A spacing is related to the purine and pyrimidine bases stacked in planes normal to the molecules. ${ }^{16}$ These X-ray results suggest that the solution has some threedimensional structure and that the arrangement of the rod-like DNA halices become more stable with the polymer concentration. At a polymer concentration of $75 \%$, the X-ray pattern shows more Debye-Scherrer rings that are sharp and suggests the formation of a crystalline (or semicrystalline) structure in accordance with the observation under crossed polaroids and the light scattering results.

The crystal of NaDNA exists in the best ordered A configuration at $75-\%$ relative humidity, and transforms to the $B$ configuration at above $80-\%$ relative humidity. ${ }^{17}$ The $\mathrm{B}$ configuration is characterized by the 3.4-A spacing. Because this spacing is observed in all the birefringent solutions tested, the double-stranded DNA molecules are considered to take the $B$ configuration in the birefringent solution. The fiber structure in the $\mathbf{B}$ configuration is semicrystalline. The double-stranded helices are expected to arrange

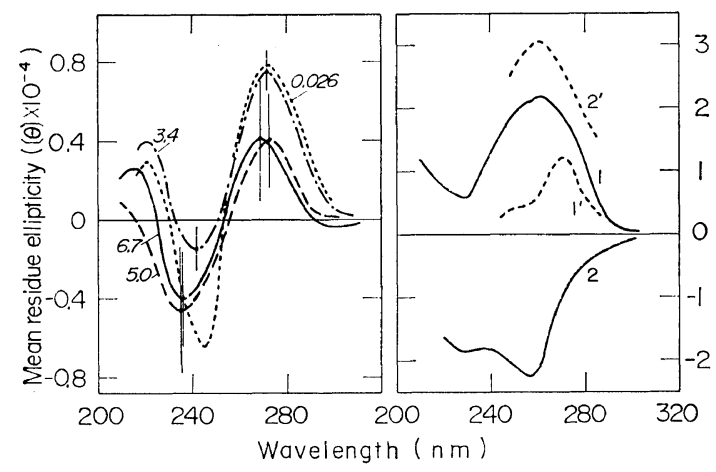

Figure 5. Circular dichroism spectra of the solutions of NaDNA. Each curve is the averaged curve. Left-hand curves, the numbers on the curves give the polymer concentration in $\%(w / w)$. Righthand curves, polymer concentration, $8.4 \%(\mathrm{w} / \mathrm{w})$; the broken curves were obtained when the optical cell was reversed. See the text.

in the hexagonal array in a molecular cluster, as in the case of PGA-Na ${ }^{5}$ or of tRNA; $;^{3}$ however, this has not been confirmed.

\section{Circular Dichroism}

The measured CD of the birefringent solution is dependent on the tilt angle of the optical cell with respect to the vertical position. If the specimen is not highly oriented or/and thin enough, the real $C D$ curve can be obtained as the average of the apparent CD curves. ${ }^{18}$ The peak and the trough of the averaged $C D$ curve of the birefringent solution make a blue shift when compared with those for the isotropic solution, and the shift is larger when the polymer concentration increases (Figure 5). Intensity of the CD becomes lower with increasing polymer concentration. This is expected when the DNA helices are uncoiled ${ }^{19,20}$ however, this is not the case here because the other results support the presence of the double-stranded helices, as has been mentioned above. Scattering or/and reflection of the incident beam due to the ordered, long-range organization in the birefringent solution would cause the decrease in the CD intensity and the modification of the features of the CD curve.

The CD curve differs from solution to solution because the fine structure of the solution is different from solution to solution even at the same polymer concentration. The difference in 
the structure increases with increasing polymer concentration and the deviation of the CD curve from the mean curve of at least eight averaged curves is larger in higher concentrations. At a polymer concentration of $8.4 \%$, the magnitude of the CD becomes even larger than when in the isotropic state and the sign of the CD often becomes opposite when the optical cell is reversed to introduce the incident beam from the opposite side of the cell surface.

\section{CONCLUDING REMARKS}

The experimental evidence reported in this study suggests, in every respect, that in solution the double-stranded NaDNA molecules aggregate to produce rod-like assemblies (molecular clusters), in which the polynucleotide molecules are aligned parallel to their long rod axes and form liquid crystals of the molecular cluster type. The polynucleotide molecules are in the B configuration, and orientation of these molecules is rather poor or/and they fluctuate in each molecular cluster. An ordered, long-range organization appears in solution and develops when the polymer concentration increases, finally leading to a (semi-?)crystalline structure. These form the principal findings in this study. The liquid crystals of NaDNA are very similar to those of deoxyHb $\mathrm{S}$ or of PGA-Na, each found in aqueous solution, and also to those of PBLG or of PELG dissolved in some organic solvents, but to a lesser extent.

The critical polymer concentration at which the birefringent phase appears (Robinson's A point $\left.{ }^{21}\right)$ is less than $1 \%(\mathrm{w} / \mathrm{w})$, as has already been mentioned. The axial ratio of the DNA double-stranded helix calculated using Flory's equation $^{22}$ then turns out to be more than 1000. This value may be reasonable if the molecular weight of the DNA used is several millions, as is often the case, and the double-stranded helix is still stiff in such a high-molecular-weight DNA. However, it may also be reasonable to say that advanced hydration around the hydrophilic groups of the polynucleotide molecules such as the phosphate groups causes the decrease of freedom of rotation of these molecules and causes the formation of liquid crystals even at such a low polymer concentration, in which the decrease of water activity accompanying the modification of the solvent $\left(\mathrm{H}_{2} \mathrm{O}\right)$ structure will be involved. In accordance with this idea, the polymer solution increases its viscosity and become gel-like when the liquid crystals are formed. This viscosity increase is common to all the aqueous liquid crystals so far mentioned. Those liquid crystals are in contrast with the usual liquid crystals, that is thermotropic liquid crystals ${ }^{23}$ and lyotropic liquid crystals of PBLG $^{24,25}$ or of PELG ${ }^{25}$ in certain organic solvents, whose, viscosities decrease markedly upon the liquid crystal formation.

Acknowledgments. I wish to thank Mr. Y. Ukai and Mr. H. Yamagishi for carrying out a part of the measurements and Dr. Y. Kondo for valuable discussions. My thanks are also due Prof. T. Samejima of Aoyama Gakuin University for valuable suggestions.

\section{REFERENCES}

1. C. Robinson, Tetrahedron, 13, 219 (1961).

2. C. Robinson, Trans. Faraday Soc., 52, 571 (1956).

3. M. Spencer, W. Fuller, M. H. F. Wilkins, F.R.S. and G. L. Brown, Nature, 194, 1014 (1962).

4. E. Iizuka, submitted for publication Proceedings of the VIth International Liquid Crystal Conference, Kent, Ohio, USA, August 1976.

5. E. Iizuka, Y. Kondo, and Y. Ukai, submitted for publication in Polym. J.

6. E. Iizuka, "Advances in Polymer Science" Vol. 20, Springer-Verlag, Berlin, 1976, pp 79-107.

7. Y. Kawada, Nippon Kagaku Zasshi (J. Chem. Soc. Japan), 78, 376 (1956).

8. J. D. Watson and F. H. C. Crick, Nature, 171, 737 (1953).

9. E. Iizuka, T. Keira, and A. Wada, Mol. Cryst., Liq. Cryst., 23, 13 (1973).

10. E. Iizuka, Polym. J., 5, 62 (1973).

11. M. B. Rhodes and R. B. Stein, J. Polym. Sci., Part A-2, 7, 1539 (1969).

12. S. F. Mason, J. Chem. Soc., 1240, 1247 (1959).

13. A. Rich and M. Kasha, J. Am. Chem. Soc., 82, 6197 (1960).

14. G. B. B. M. Sutherland and M. Tsuboi, Proc. Roy. Soc. (London), A239, 446 (1957).

15. E. Iizuka, unpublished data.

16. W. T. Astbury, Symp. Soc. Exp. Biol., I. Nucleic Acid, University Press, Cambridge, 1947, p 66. 
E. IIzUKA

17. R. E. Franklin and R. G. Gosling, Acta Crystallogr., 6, 673 (1953).

18. M. J. B. Tunis-Schneider and M. F. Maestre, J. Mol. Biol., 52, 521 (1970).

19. J. Brahms and W. F. H. M. Mommaerts, ibid., 10, 73 (1964).

20. T. Samejima and J. T. Yang, J. Biol. Chem., 240, 2094 (1965).

21. C. Robinson, J. C. Ward, and R. B. Beevers,
Disc. Faraday Soc., 7, 775 (1958).

22. P. J. Flory, Proc. Roy. Soc. (London), A234, 73 (1956).

23. G. W. Gray, "Molecular Structure and the Properties of Liquid Crystals," Academic Press, London, 1962, Chapter 6.

24. J. Hermans, Jr., J. Colloid Sci., 17, 638 (1962).

25. E. Iizuka, Mol. Cryst. Liq. Cryst., 25, 287 (1974). 